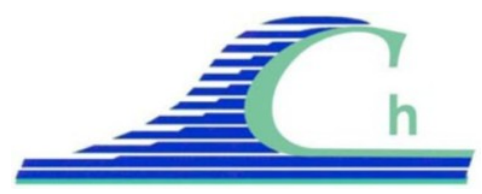

XII ${ }^{\text {ìmes }}$ Journées Nationales Génie Côtier - Génie Civil

Cherbourg, 12-14 juin 2012

DOI:10.5150/jngcgc.2012.098-B @ Editions Paralia CFL

disponible en ligne - http://www.paralia.fr - available online

\title{
Prévision des impacts des tempêtes : exemple du démonstrateur MICORE sur le Lido de Sète
}

\section{Rémi BELON $^{1}$, Yann BALOUIN ${ }^{2}$, Jérôme THIEBOT ${ }^{3}$, Etienne DELVALLE ${ }^{3}$, Déborah IDIER $^{3}$, Rodrigo PEDREROS ${ }^{3}$}

1. BRGM, Service Géologique Régional, Immeuble Agostini Z.I de Furiani, 20600 Bastia, France,

r.belon@brgm.fr

2. BRGM, Service Géologique Régional, 1039 rue de Pinville, 34000 Montpellier,

France.

3. BRGM, Service Risque Côtier, Avenue Claude Guillemin, 45060 Orléans, France.

\section{Résumé :}

Ces dernières années, les événements hydrométéorologiques extrêmes en zone côtière ont souligné les effets dévastateurs que peuvent entrainer les aléas côtiers maritimes et mis en évidence la nécessité de pouvoir anticiper les phénomènes météo-marins et leurs impacts. Dans ce contexte, l'objectif principal du projet européen MICORE (Morphological Impacts and COastal Risks induced by Extreme storm events) était, dans les 9 pays participants, de mettre en place et de tester un système d'alerte en ligne pour la prévision des impacts morphologiques dus aux tempêtes marines.

Le prototype de système d'alerte repose sur une structure générique commune aux différents littoraux étudiés qui est basée sur 5 modules : un module d’observation, un module de prévision, un module d'aide à la décision, un module d'alerte et un module de visualisation. Le prototype développé sur le littoral du Lido de Sète exécute quotidiennement ces différents modules.

Le module de prévision à 3 jours, au cœur du système est constituée d'une chaine de modèles partant de la prévision opérationnelle météo-océanographique disponible, la propagation de l'hydrodynamique à la côte, et la modélisation morphodynamique sur la plage en utilisant le modèle open-source X-Beach qui a été préalablement validé sur le site du Lido de Sète en terme d'évolution morphologique et d'hydrodynamique. Les sorties du modèles X-Beach sont traduites en SII (Storm Impact Indicators), indicateurs dérivés permettant la prise de décision par les gestionnaires.

La présente contribution présentera le prototype de système d'alerte, sa validation, et les applications proposées sur le Lido de Sète en termes de protection civile et d'anticipation des submersions par franchissement du cordon dunaire.

\section{Mots-clés :}

Océanographie opérationnelle - Situations extrêmes - Hydrodynamique - prévision météorologique 


\section{Introduction}

Ces dernières années, les évènements hydrométéorologiques extrêmes en zones côtières ont souligné les effets dévastateurs que peuvent entrainer les aléas côtiers maritimes. En effet, des évènements tels que la tempête de 1953 qui créa l'une des inondations la plus importante des Pays-Bas, ou encore plus récemment l'ouragan Katrina qui a frappé la ville de la Nouvelle-Orléans et la tempête Xynthia qui toucha plusieurs pays européens nous rappellent à quel point nos côtes peuvent être vulnérables à ces phénomènes.

D’un point de vue économique, il est difficile de concevoir des solutions permettant de protéger l'ensemble de nos côtes. A cela se rajoute l'impact du changement climatique qui introduit de nouvelles incertitudes sur ce type d'évènements en termes de fréquence d'apparition, d'intensité... Il est donc important de développer notre capacité à prédire non seulement l'arrivée imminente de ces phénomènes mais également les impacts potentiels que peuvent avoir ces évènements sur notre littoral afin d'anticiper les mesures appropriées afin de limiter le risque pour la population. Dans ce contexte, l'objectif principal du projet européen MICORE (Morphological Impacts and COastal Risks induced by Extreme storm events FP7 n²02798, 2008-2011) était, dans les 9 pays participants, de mettre en place et de tester un système d'alerte en ligne pour la prévision des impacts morphologiques dus aux tempêtes.

\section{Le projet MICORE}

Le projet MICORE est une initiative européenne associant 16 institutions de recherche, entreprises et gouvernements de 9 pays. L’objectif principal du projet était de mettre en place et tester un système d'alerte en ligne pour la prévision des impacts morphologiques dus aux tempêtes marines en soutien aux stratégies de protection civile (CIAVOLA et al., 2011).

Le projet s'est focalisé sur 9 sites-pilotes. Le choix de ces sites aux caractéristiques très variables permet de développer une approche aussi générique que possible et de démontrer la robustesse de la méthodologie. Chacun de ces sites a fait l'objet de plusieurs phases de travaux dans le but de mettre en place un système d'alerte. 5 phases ont ainsi été réalisées : (1) Une revue des évènements historiques ; (2) Un suivi sur le terrain des évènements de tempêtes; (3) L’utilisation des données de terrain pour évaluer et valider les modèles existants ainsi qu'un nouveau modèle développé pour les impacts des tempêtes ; (4) Le développement d'un prototype de système d'alerte ; (5) Le lien entre le système d'alerte et les protocoles de protection civile.

Les prévisions météorologiques étant généralement fournies à 3 jours, le projet MICORE vise plus à développer des outils de réponse à court-terme que des stratégies de gestion à long-terme. 9 démonstrateurs ont été développés dans le cadre du projet, et cette contribution présente l'exemple du démonstrateur sur le Lido de Sète. 


\section{XII ${ }^{\text {èmes }}$ Journées Nationales Génie Côtier - Génie Civil \\ Cherbourg, 12-14 juin 2012}

\section{Description du site}

Le Lido de Sète est situé entre Sète et Marseillan dans la région du LanguedocRoussillon dans le Sud de la France. Le lido de Sète est une barrière de sable étroite séparant la mer méditerranée de l'étang de Thau. De nombreuses activités développées sur ce territoire telles que l'hôtellerie, la viticulture, les installations touristiques ainsi que les activités de pêche dans le lagon sont des activités liées à la «bonne santé » du littoral et peuvent entraîner de graves conséquences économiques, sociales et environnementales si elles sont amenées à disparaître. Les enjeux touristiques et économiques sont donc particulièrement importants.

Le Lido de Sète est un système côtier typique du littoral méditerranéen. La morphologie est caractérisée par une plage étroite et basse et des morphologies de barres d'avant-côte interne et externe qui sont pseudo-rectilignes à rythmiques (CERTAIN, 2002). En 2008, un grand programme de ré-aménagement du site a été initié par Thau Agglo. Le projet visait notamment à relocaliser la route nationale (souvent détruite par les tempêtes hivernales) et à reconstruire une plage et un cordon dunaire «naturels ». Après travaux, la plage mesure environ $70 \mathrm{~m}$ de large, et le cordon dunaire, stabilisé par des ganivelles, atteint 3 m NGF.

Sur ce littoral, les principaux impacts des tempêtes marines concernent l'érosion de la plage, l'érosion du cordon dunaire (et auparavant de l'ex-route nationale qui était implantée sur la dune), le franchissement du cordon et l'inondation résultante. Ce sont donc ces effets des tempêtes que le projet MICORE a particulièrement étudié pour le développement d'un prototype de systèmes d'alerte des impacts morphologiques.

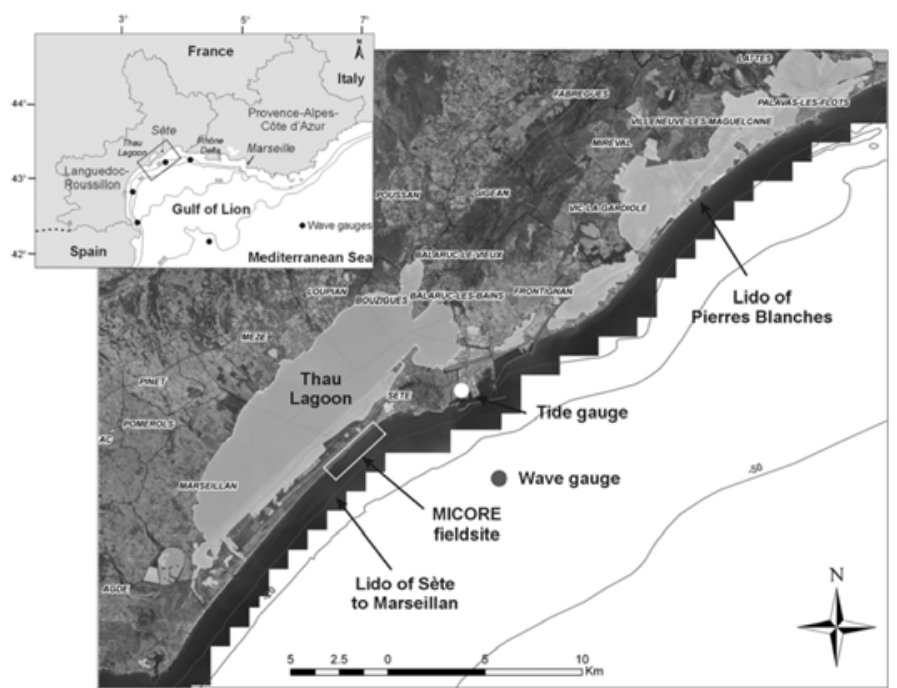

Figure 1. Localisation du site. 


\section{Description du prototype}

Le prototype de système d'alerte repose sur une structure générique commune aux différents littoraux étudiés qui est basée sur 5 modules : un module d'observation, un module de prévision, un module d'aide à la décision, un module d'alerte et un module de visualisation. Le prototype développé sur le littoral du Lido de Sète exécute quotidiennement ces différents modules.

\section{a) Le module d'observation :}

Ce module concerne à la fois les observations hydrométéorologiques au travers de la collecte des données météorologiques (vent, pression) mais également au travers des mesures de la houle, des niveaux d'eau (marée, surcote) ainsi que des observations morphologiques telles que les profils de plage.

b) Le module de prévision :

Ce module consiste à prédire les caractéristiques des tempêtes à la côte notamment en terme de niveau d'eau atteint sur la plage durant l'évènement prévu. Le modèle de prévision météorologique AROME de Météo France n’étant pas encore disponible, il a été choisi d'utiliser le modèle de prévision GFS pour obtenir les champs de vent et de pression sur la zone d'étude. Ces données sont ensuite introduites dans le modèle MARS de l'Ifremer (LAZURE \& DUMAS, 2007) afin de calculer le niveau d'eau sur notre zone d'étude. Les prévisions de la houle obtenues dans le cadre de Previmer (www.previmer.org) sont extraites au large et sont propagées jusqu'à notre profil de plage par le modèle SWAN (BOOIJ et al., 1999). Toutes ces données sont alors introduites dans le modèle Xbeach (ROELVINK et al., 2009) afin d'en extraire les répercussions sur le profil de plage.

\section{c) Le module d'aide à la décision :}

Les paramètres physiques tels que les volumes érodés sur les dunes, les vitesses de courant, les niveaux de run-up, les débits franchissant sont couramment utilisés par les experts pour quantifier les impacts des tempêtes. Cependant, du point de vue d'un utilisateur final, ces paramètres sont difficiles à utiliser de manière opérationnelle, lorsque des décisions rapides doivent être prises sur la base des informations disponibles. Les modules de prévision et de décision du système d'alerte se sont par conséquent attachés au développement des dits Indicateurs d'Impact des Tempêtes (SII). Les SII sont une quantification du système côtier adaptés à la prise de décision. Des niveaux de seuils leurs sont associés, déterminant les niveaux d'action à mettre en œuvre par les autorités. 


\section{XII ${ }^{\text {èmes }}$ Journées Nationales Génie Côtier - Génie Civil \\ Cherbourg, 12-14 juin 2012}

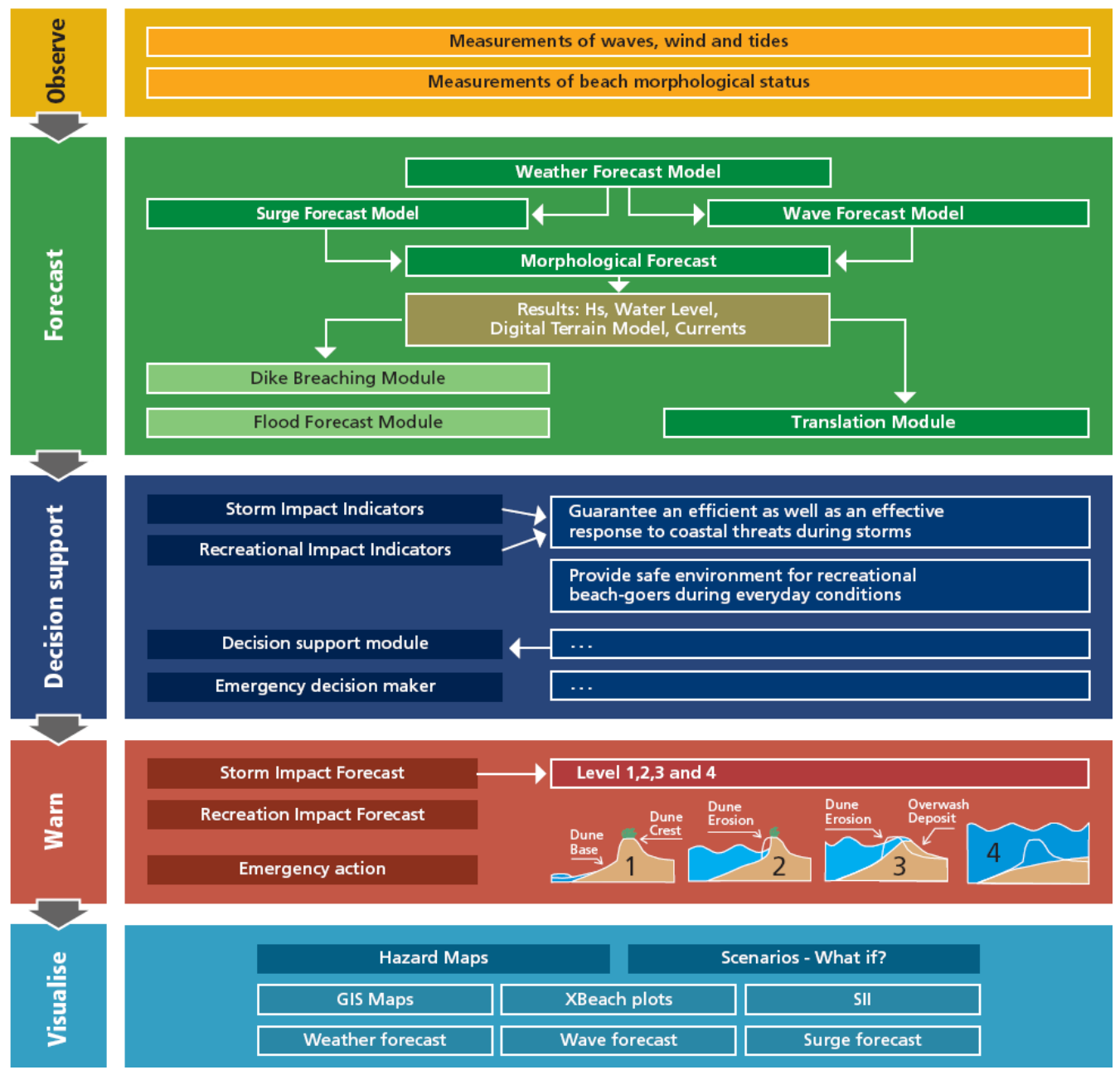

Figure 2. Présentation des différents modules du prototype.

Sur le Lido de Sète, le prototype de système d'alerte est basé sur deux indicateurs d'impacts des tempêtes: (1) l'Indicateur de Préparation à l'évacuation, lié au risque de franchissement du cordon dunaire, et à l'inondation résultante (EPI) ; et (2) l'Indicateur de sécurité côtière lié à la sécurité des personnes présentes sur le littoral (CSI). Un seul paramètre sera pris en compte à savoir le niveau d'eau (marée prédite, surcote atmosphérique, set-up et run-up) atteint sur la plage qui sera comparé avec la morphologie de la plage afin de définir si le niveau présent sur la plage présente un risque ou non pour ces 2 indicateurs. Ce paramètre permet de refléter l'impact direct que peut avoir la houle sur la plage.

\section{d) Le module d'alerte :}

Pour ces 2 indicateurs définis ci-dessus, des niveaux d'alerte ont été définis. Pour le cas de l'EPI, on considère que le risque est présent à partir du moment où une couche d'eau de $50 \mathrm{~cm}$ est présente sur la plage. La morphologie de la plage nous incite donc à définir ces 3 niveaux d'alerte (cf. table 1 et figure 4). Pour l'indicateur CSI, le niveau d'eau est 
comparé à la morphologie de la dune, ainsi dès que le niveau d'eau atteint à minima le pied de dune alors la notion de risque apparaît. Trois niveaux d’alerte sont définis.

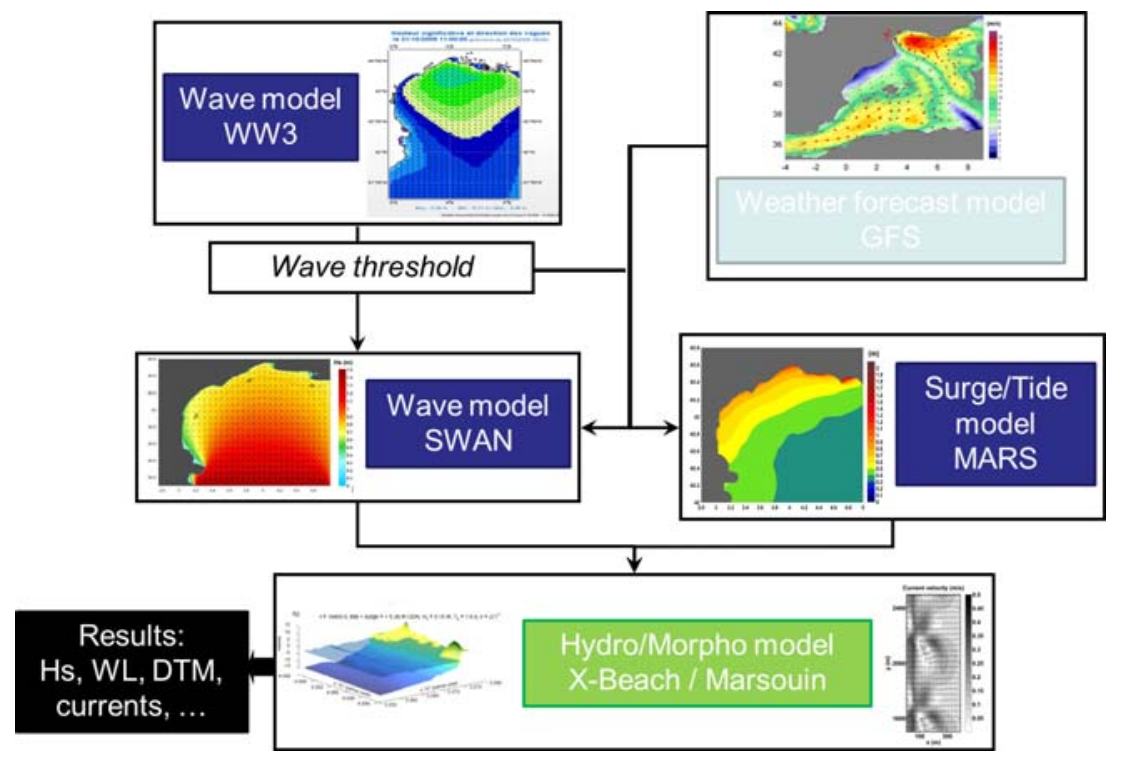

Figure 3. Chaînage des modèles en vue d'obtenir les prévisions.

e) Le module de visualisation :

Les niveaux de risques associés à chaque indicateur (rouge $=$ fort ; orange $=$ modéré ; vert=faible) sont calculés quotidiennement sur des segments de plage et simplement représentés par des lignes parallèles sur une carte interactive google map. De cette manière, les autorités en charge de la protection civile peuvent facilement et rapidement visualiser le danger que peuvent encourir les usagers du site.
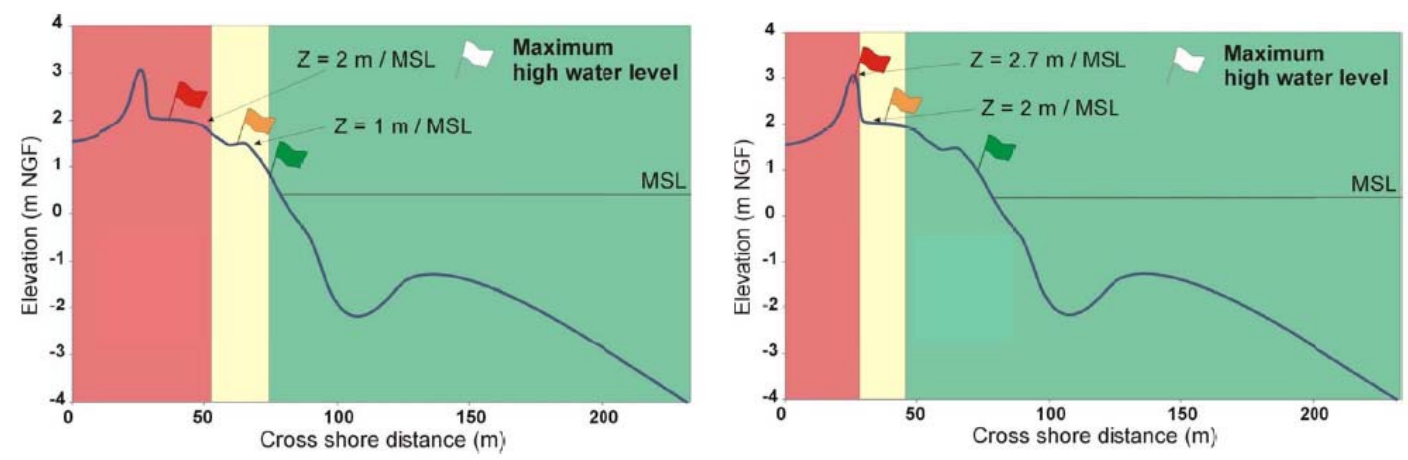

Figure 4. Les indicateurs retenus sur le Lido de Sète et leurs seuils :EPI (à gauche) et CSI (à droite). 


\section{XII ${ }^{\text {èmes }}$ Journées Nationales Génie Côtier - Génie Civil \\ Cherbourg, 12-14 juin 2012}

\section{La validation des modèles}

Pour que ce prototype de système d'alerte soit réaliste, une phase de validation a été réalisée. Lors des deux hivers successifs (2008-2010), une série de mesures prétempêtes et post-tempêtes a été réalisée pour chaque évènement significatif. Le modèle n'étant pas apte à simuler correctement la dynamique complexe des barres d'avant-côte, la validation présentée ici concerne la simulation de l'hydrodynamique par le prototype. Cette phase de validation est double. Elle concerne d'une part les niveaux d'eau (surcote) obtenus grâce au modèle Mars (Ifremer) utilisant les prévisions météorologiques GFS, qui sont comparés avec les mesures du marégraphe de Sète, d'autre part, les niveaux d'eau liés au set-up des vagues simulés avec Xbeach et comparés au mesures du marégraphe de Sète. Les données de houle forçant la chaine de modèles sont issues des simulations de WW3 (Previmer).

Modèle Mars et prévisions GFS: A titre d'exemple, la Figure 5 présente la comparaison des observations in-situ (marégraphe) et du modèle entre le 7 septembre 2011 à 0 h et le 13 septembre 2011 à 23h30. La corrélation entre les simulations et les mesures est tout à fait satisfaisante, avec un $\mathrm{R}^{2}=89.7 \%$.

L'effet du set-up calculé par Xbeach : Entre Nov. 2008 et Mars 2009, la plus forte hauteur significative de la houle fut atteinte lors de la tempête du 26 décembre 2008.

Les caractéristiques de cet événement sont résumées dans la Table 2. La Houle atteignit la valeur de $4 \mathrm{~m}$ au large du Lido de Sète. Le niveau d'eau moyen (port de Sète) était de l'ordre de $40 \mathrm{~cm}$ par rapport au Zéro Hydrographique (le niveau des plus basses mers astronomiques) avec une surcote de $25 \mathrm{~cm}$.

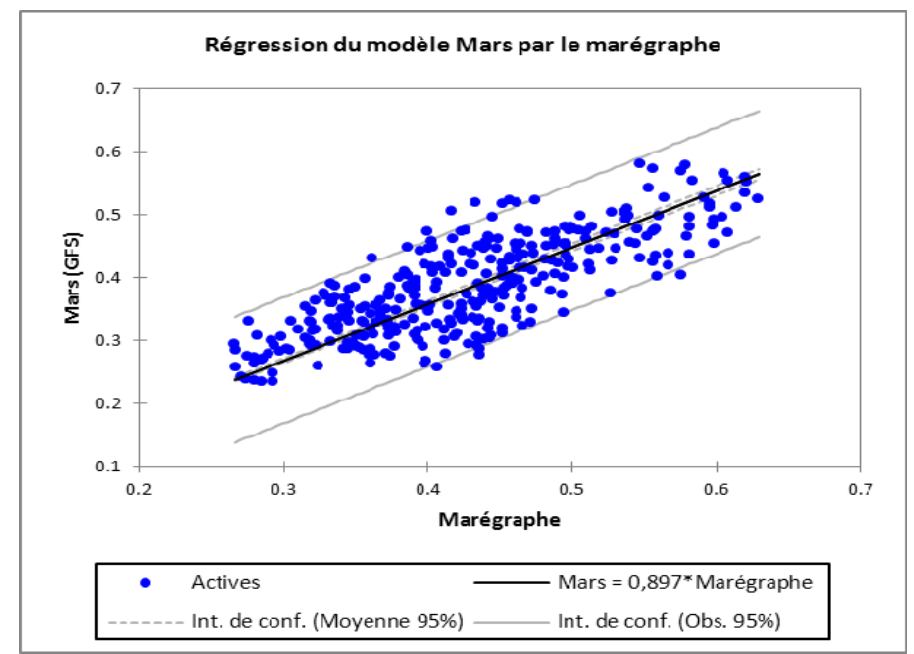

Figure 5. Comparaison entre les prévisions du modèle Mars (GFS) et le marégraphe de Sète. 
Thème 7 - Océanographie opérationnelle et situations extrêmes

Table 1. Résumé des caractéristiques de la tempête

\begin{tabular}{|c|c|c|c|c|c|c|c|}
\hline $\begin{array}{l}\text { Date du pic } \\
\text { de tempête }\end{array}$ & Hs max & $\begin{array}{l}\text { Ts à } H s \\
\max \end{array}$ & $\begin{array}{l}\text { direction } \\
\text { quand } \\
\text { Hs max }\end{array}$ & Incidence & $\begin{array}{l}\text { Niveau } \\
\text { d'eau } \\
\text { quand } \\
\text { Hs max }\end{array}$ & durée & Energie \\
\hline date & $m$ & $s$ & ${ }^{\circ} N$ & $\circ$ & $m$ & $h$ & $M W$ \\
\hline 26/12/08 18h & 3.99 & 10 & 121.12 & -17 & 0.65 & 140 & 8.014 \\
\hline
\end{tabular}

Les résultats obtenus avec le modèle Xbeach montrent la bonne aptitude du modèle à reproduire l'effet du set-up dû aux vagues dans la zone de déferlement qui atteint pour cette tempête une valeur proche de $20 \mathrm{~cm}$ (Figure 6 et Figure 7). Pour rappel, le set-up est la surélévation du plan d'eau dû au déferlement de la houle. Cette surélévation dépend à la fois de la hauteur significative de la houle à l'approche de la côte ainsi que de la morphologie de l'avant-côte.

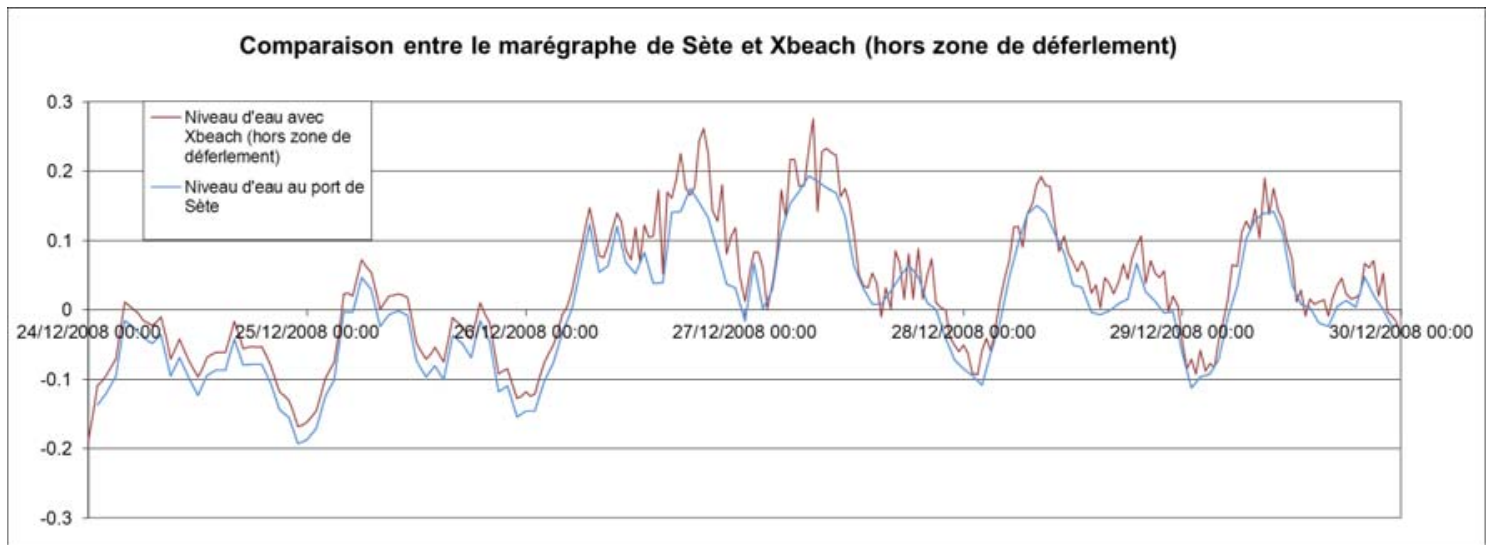

Figure 6. Comparaison des niveaux d'eau totaux (m NGF) entre le modèle Xbeach (hors zone de déferlement) et le marégraphe de Sète.

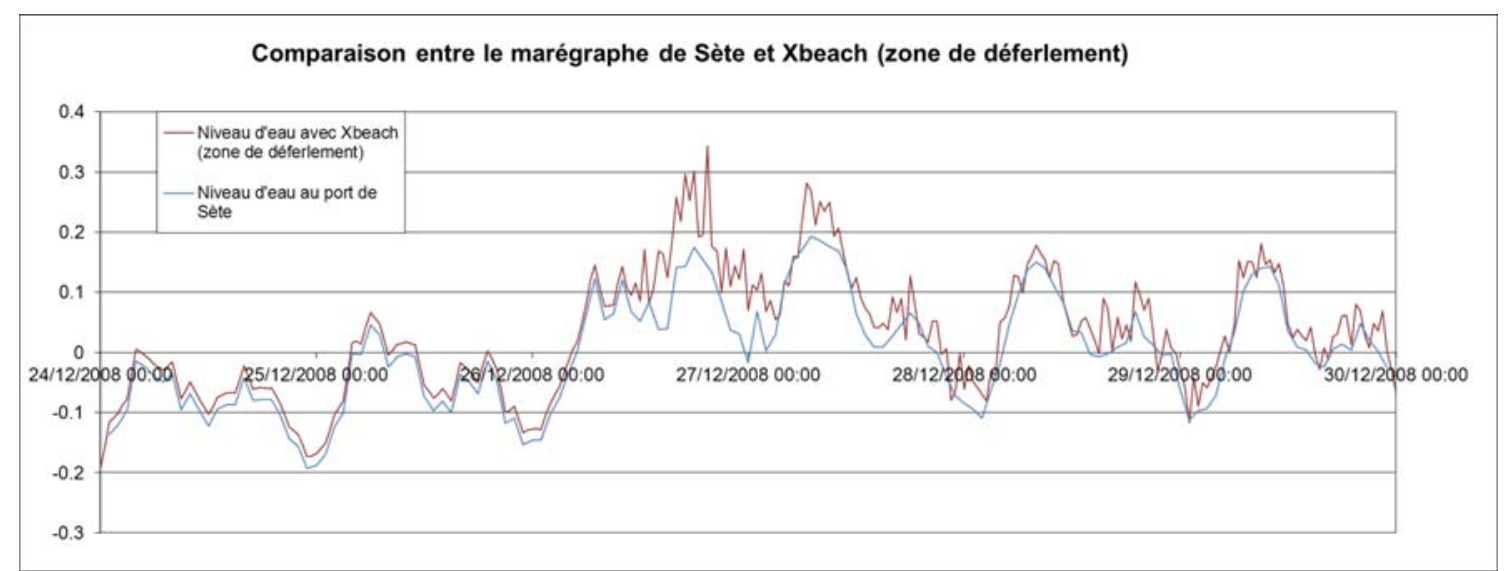

Figure 7. Comparaison des niveaux d'eau totaux (m NGF) entre le modèle Xbeach (zone de déferlement) et le marégraphe de Sète. 


\section{XII ${ }^{\text {èmes }}$ Journées Nationales Génie Côtier - Génie Civil \\ Cherbourg, 12-14 juin 2012}

Cette validation mériterait d'être approfondie en utilisant notamment des moyens de mesures dans la zone de déferlement (capteur de pression) ainsi que des mesures de runup (jet de rive) afin d'évaluer la précision des niveaux obtenus par le modèle sur la plage. L'utilisation du marégraphe de Sète est limitée en raison du fait que l'effet du setup n'est pas mesuré.

\section{Conclusion}

$\mathrm{Vu}$ le contexte actuel du réchauffement climatique et la difficulté que nous avons de pouvoir anticiper les évolutions en termes de périodicité et d'intensité des évènements tempétueux à venir, il est important de développer des outils qui pourront permettre de mieux appréhender ces phénomènes.

C'est dans ce cadre que vient s'inscrire ce démonstrateur de système d'alerte sur le Lido de Sète. Cet article présente la faisabilité et la cohérence des résultats hydrodynamiques d'un tel système.

La phase de validation qui mériterait d'être approfondie contribue à l'évaluation des modèles hydrodynamiques tels que MARS, SWAN ou XBEACH à partir des données de prévisions météorologiques GFS. La validation de l'effet du set-up ainsi que du runup permettrait une meilleure appréhension des résultats obtenus et ainsi de réaliser un calage précis des modèles.

Le prototype a montré sa capacité à prédire des niveaux d'eau cohérents sur le littoral du Lido de Sète, et peut donc être utilisé pour calculer les indicateurs de tempêtes et fournir les éléments relatifs à la gestion de risques côtiers liés aux événements de tempêtes marines.

\section{Remerciements}

Ce travail a été réalisé en partie dans le cadre du projet FP7 MICORE n²02798

\section{Références bibliographiques}

BOOIJ N., RIS R.C., HOLTHUIJSEN L.H. (1999). A third-generation wave model for coastal regions, Part I, model description and validation. Journal of Geophysical Research, C4 (104), pp 7649-7666. doi:10.1029/98JC02622

CERTAIN R. (2002). Morphodynamique d'une côte sableuse microtidale à barres : le Golfe du Lion (Languedoc - Rousillon). Thèse de doctorat Université de Perpignan, 209 p.

CIAVOLA P., FERREIRA O., HAERENS P., VAN KONINGSVELD M., ARMAROLI C. (2011). Storm impacts along European coastlines. Part 2: lessons learned from the MICORE project. Environmental Science \& Policy, Vol. 14(7), pp 924-933. doi:10.1016/j.envsci.2011.05.009 
Thème 7 - Océanographie opérationnelle et situations extrêmes

LAZURE P., DUMAS F. (2007). An external-internal mode coupling for a 3D hydrodynamical model for applications at regional scale (MARS). Advances in Water Resources, Vol. 31, pp 233-250. doi:10.1016/j.advwatres.2007.06.010

ROELVINK D., RENIERS A., VAN DONGEREN A., VAN THIEL DE VRIES J., McCALL R., LESCINSKI J. (2009). Modelling storm impacts on beaches, dunes and barrier islands. Coastal Engineering, Vol. 56 (11-12), pp 1133-1152. doi:10.1016/j.coastaleng.2009.08.006 